\title{
DESIGN OF A MICROCHANNEL BASED SOLAR RECEIVER/REACTOR FOR METHANE-STEAM REFORMING
}

\author{
Drost, K. J., Eilers, B., Apte, S.V., Narayanan, V.† and Schmitt, J. \\ School of Mechanical Industrial and Manufacturing Engineering \\ Oregon State University, 204 Rogers Hall, Corvallis, OR 97331
}

\begin{abstract}
This study investigates use of solar thermochemical processing of clean fuels using biomass products (in particular $\mathrm{CH}_{4}, \mathrm{H}_{2} \mathrm{O}$ ). To address technological feasibility of a microchannel-based solar receiver/reactor, a combined numerical and experimental study of methane-steam reforming is carried out on a single microchannel with Palladium-deposited channel walls and heat input to facilitate endothermic heterogeneous reactions producing hydrogen. A simple one-dimensional model solving steady state species mass fraction, energy, and overall conservation of mass equations is developed, calibrated and validated against concurrent experimental data [1,2]. Methane-steam reforming is modeled by three reduced-order reactions occurring on the reactor walls. The effects of the total heat input, heat flux profile, and inlet flow rate on production of hydrogen are investigated to assess the effectiveness of the microchannel configuration for production of hydrogen. A coupled shape-constrained optimization and Monte-Carlo radiative heat transfer model is developed to design a receiver shape that can yield a desired heat flux distribution on the channel walls for improved yield of hydrogen.

*sva@engr.orst.edu

†narayavi@engr.orst.edu
\end{abstract}




\section{INTRODUCTION}

Solar energy is a promising clean energy source that is of great economic and technological importance. Typically, one of three conversion pathways are followed in converting solar energy to usable energy: (a) direct electrical power through photovoltaics, (b) solarthermal electric power generation, or (c) chemical processing and production of hydrogen or other fuels $[3,4,5,6]$. The present work is motivated from the perspective of trying to address important technological challenges that could enable efficient solar thermochemical processing of clean fuels using biomass products $\left(\mathrm{CO}_{2}, \mathrm{CH}_{4}\right)$.

The technological readiness of solar concentrators is relatively high [6, 7]; however, there are several areas for advancement in receiver technology that are needed for improving solar thermochemical processes. Receiver designs that permit increase in the overall solar to chemical conversion efficiency are needed. Such designs would necessitate reductions in convective and re-radiative losses from the receiver. The reactor, within which chemical processes take place, needs to permit high completion rate of endothermic reactions. In addition to the receiver and reactor design, process control schemes that could optimize the production of fuels is needed. Solar receivers based on a volumetric or cavity receiver design are common [8, 9, 10], wherein chemical reactions occur in cylindrical cavities by constraining the reactants and products within the cavity. Chemical reactions in such receivers could be limited by the diffusion time of the non-premixed reactants, low heat transfer rates from the walls to the reactants, and low volumetric absorption by the reactant gases.

The present work investigates conversion efficiency of biomass-based gaseous products (in particular $\mathrm{CH}_{4}$ ) using a microchannel-based reactor, as shown in Figure 1. The concept would involve an array of several microchannel reactors shaped to form the receiver. This design exploits the short diffusion lengths for reactant gases in microchannels, such that the reaction may occur near stoichiometric conditions (using very less excess reactant gases), thereby substantially increasing the efficiency of the system. In order to study the technical feasibility of such a design, numerical simulations and modeling are used to investigate the strong endothermic reactions of methane-steam reforming inside a microchannel reactor with Palladium catalyst. The simulations are calibrated and validated against concurrent experiments [1]. Previous work [11] also involved detailed numerical simulation using a low-Mach number, unsteady, variable-density Navier-Stokes

equations together with species mass-fraction and energy equations solved for a three-dimensional microchannel configuration. 


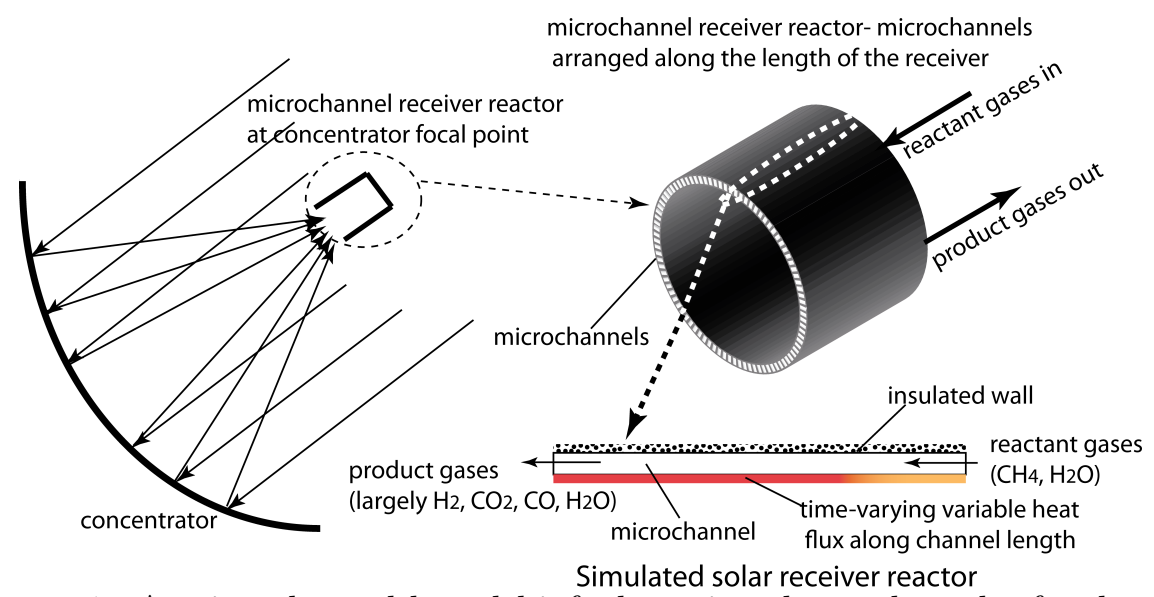

Figure 1: A microchannel-based biofuel receiver located at the focal point of a parabolic solar concentrator. The shape of the receiver, which is comprised of multiple microchannel reactors, is obtained from shape-constrained optimization. A simulated large aspect ratio microchannel reactor is used in the present study.

The primary objective of the present work is to develop a simple onedimensional model for the thermochemical processes inside the reactor. The main reason to develop such model is to facilitate several parametric studies that can be used to obtain operation range as well as identify conditions for improved hydrogen production.

The second objective of this work is to develop methodology that will provide the shape of the receiver necessary to obtain a desired heat flux distribution along the microchannel walls. Specifically, knowing the heat flux distribution that produce improved hydrogen production, the goal is to design the receiver shape that will yield such a distribution. This requires solution of the radiative heat transfer problem (done using the MONT2D software [12]) that provides shape factors for a chosen receiver geometry. Next, the radiative solver is coupled with a shape-constrained optimization procedure to obtain a receiver shape that will closely provide the desired heat flux distribution through an iterative approach.

The paper is arranged as follows. A brief description of the experimental setup and the computational model is given first. The mathematical formulation for heterogeneous surface reaction kinetics as well as the onedimensional model are summarized next. A brief description of the numerical approach is given next. Calibration and validation of the model are presented followed by parametric studies using the one-dimensional model. 
Finally, the shape-constraint optimization procedure coupled with a radiative heat transfer algorithm is described next.

\section{EXPERIMENTAL SETUP}

The experimental setup for the present study corresponds to a single large aspect ratio microchannel considered by Eilers [1] as shown in figure 2a. Heat input through bottom wall of the microchannel was provided by propane burners via direct flame impingement, simulating solar energy flux. The propane burners could be controlled individually to provide a spatial variations in heat flux along the reactor. The reactor had nine segments of controlled heat input with $0.8 \mathrm{~mm}$ gaps between each segment to minimize substrate conduction between segments. The first two segments were for preheating the reactant gases while a porous catalyst bed formed the bottom wall in the remaining seven heated segments. The microchannel was formed by a high temperature gasket shim, compressed between two pieces of stainless steel to form a large aspect ratio channel. The dimensions of the microchannel were $173.5 \mathrm{~mm}$ in length, $19 \mathrm{~mm}$ in width, and 700 micrometers in height. Palladium catalyst was contained on a porous FeCrAlY felt insert that was $133.5 \mathrm{~mm}$ long.

The channel was held together with 30 stainless steel bolts distributed around the perimeter of the channel in order to distribute the compressive force and to minimize the potential for leaks in the system.

(a)

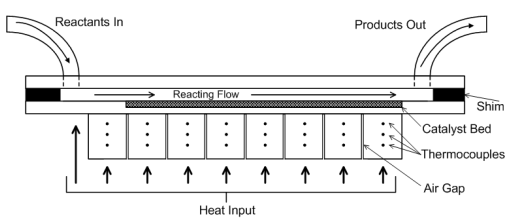

(b)

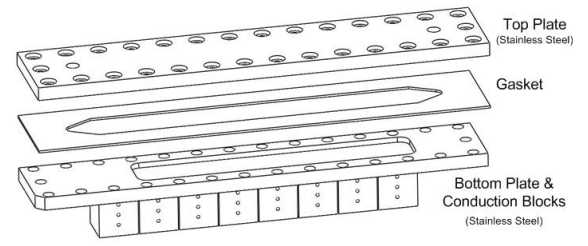

Figure 2: Large-aspect ratio microchannel solar reactor $[1,2]$. (a) Schematic of the reactor, (b) expoded view of the reactor.

The catalyst bed was formed of a FeCrAlY sheet that was oxidized in a furnace at $900 \mathrm{C}$ for four hours in an air environment, to create $\alpha$ alumina and increase the surface area for catalyst deposition. Palladium nano-particles were synthesized using a modified form of the methods proposed in references $[13,14]$. The bed was dip coated in the $\mathrm{Pd}$ nanoparticle solution in toluene using a selective technique to enhance the percentage of catalyst present at the surface of the catalyst bed. Details of the catalyst 
preparation and deposition are given in Eilers [1]. Figure 3 shows a sample SEM image of the catalyst bed after flow shearing test. Particle sizes on the order of $15 \mathrm{~nm}$ were observed but a large degree of agglomeration occurred as can be seen in figure 3. No noticeable reduction in particle density was observed after the shear testing, suggesting that particles were adequately adhered to the substrate.

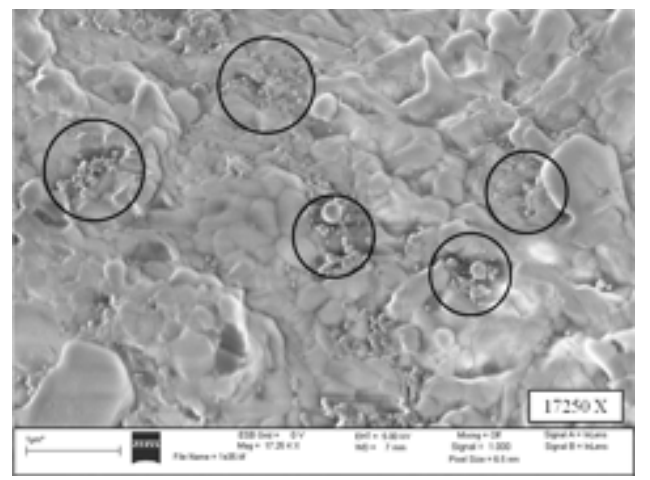

Figure 3: SEM image of the catalyst bed after shear testing [1].

Parametric studies of steam-methane ratio, residence time, average reactor temperature, and temperature distribution were performed experimentally; details are reported in Eilers et al. [1,2]. Methane conversion was found to be strongly dependent on reactor temperature. Linear ramp temperature distributions demonstrated about a 46 percent greater hydrogen output than isothermal reactions performed at the same average temperature. Results from the experiments were used to calibrate the reaction rate constant in the 1-D numerical simulations described below.

\section{MATHEMATICAL FORMULATION}

To perform parametric studies and geometric optimization, a simplified onedimensional reacting flow model for steady state solution was developed. Accordingly, the plane section of the microchannel (neglecting the inlet and outlet pipe sections in Figure 2a) was considered with one-dimensional grid along the flow direction. Using a simple staggered grid and neglecting diffusion in the wall-normal direction, the following steady state equations can be derived: 


$$
\begin{aligned}
\frac{d}{d x}(\rho \bar{u}) & =\frac{d}{d x}\left(\dot{m}^{\prime \prime}\right)=0 \\
\frac{d}{d x}\left(\dot{m}^{\prime \prime} Y_{i}\right) & =\frac{d}{d x}\left(\rho \mathcal{D}_{i, m} \frac{d Y_{i}}{d x}\right)+\dot{\omega}_{i}^{\prime \prime \prime}+\dot{s}_{i}^{\prime \prime \prime} \\
\frac{d}{d x}\left(\dot{m}^{\prime \prime} h_{s}\right) & =-\frac{d \dot{q}^{\prime \prime}}{d x}-\sum_{i}^{N_{s}} \dot{\omega}_{i}^{\prime \prime \prime} h_{f_{i}}^{o}+\dot{q}_{\mathrm{surf}}^{\prime \prime \prime}
\end{aligned}
$$

where $\bar{u}$ is the average velocity over the cross-section of the microchannel, $N_{s}, \rho, Y_{i}, h_{s}=\int_{T_{r e f}}^{T} c_{p_{m i x}} d T$, and $\dot{q}^{\prime \prime}$, represent the number of species transport equations, density, species mass fraction, mixture specific enthalpy, and heat flux due to conduction and species diffusion, respectively. The mixture is assumed as an ideal gas with the viscosity, thermal conductivity, and the binary diffusion coefficient $\left(\mathcal{D}_{i, m}\right)$ depending upon the local composition and temperature. Also $\dot{\omega}_{i}^{\prime \prime \prime}$ represents source terms corresponding to the homogeneous gas-phase reactions in the species mass fraction. Corresponding source in the energy equation is based on the species heat of formation, $h_{f_{i}}^{o}$. The mixture density is obtained from the ideal gas equation,

$$
\begin{aligned}
p & =\frac{\rho R T}{\mathcal{M}_{\text {mixture }}} \\
\mathcal{M}_{\text {mixture }} & =\frac{1}{\sum_{i}^{N_{s}} \frac{Y_{i}}{\mathcal{M}}{ }_{i}}
\end{aligned}
$$

, where, $R$ is the universal gas constant, $p$ is the operating pressure, $T$ is temperature, $\mathcal{M}_{\text {mixture }}$ is the mixture molecular weight, and $N_{s}$ is the total number of species. Thermodynamic pressure inside the microchannel is assumed constant in this low-Mach number formulation.

The majority of chemical conversion takes place on the catalyst surface giving rise to heterogeneous reactions modeled by the three surface reactions described in the next section. Since a one-dimensional axial model is considered in the present work, the surface reactions and species generation/destruction are implemented as additional source terms in the species transport $\left(\dot{s}_{i}^{\prime \prime \prime}\right)$ and energy equations $\left(\dot{q}_{\text {surf }}^{\prime \prime \prime}\right)$. Using a finite volume approach and integrating the one-dimensional model over the control volume, implementation of the surface reactions and surface heat flux into this model is fairly straight forward. The expressions for these source terms are described below. 
The catalytic conversion occurring on the reactor surface can be formulated as

$$
-\rho \mathcal{D}_{i m} \frac{\partial Y_{i}}{\partial n}=\dot{s}_{i}^{\prime \prime} \mathcal{M}_{i} ; \quad \dot{q}_{\text {surf }}^{\prime \prime}=\dot{q}_{\text {wall }}^{\prime \prime}+\frac{1}{A_{\text {wall }}} \sum_{k}^{3} r_{k} \Delta H_{R, k}^{0},
$$

where $\dot{s}_{i}^{\prime \prime}$ is the rate of chemical species adsorption and desorption at the catalyst surface, $\mathcal{M}_{i}$ is the molecular weight of species $i, \dot{q}_{\text {wall }}^{\prime \prime}$ is the rate of external heat supplied to the wall, $\Delta H_{R, k}^{0}$ is the heat of reaction, $r_{k}$ is the reaction rate of the $k^{\text {th }}$ surface reaction, and $A_{\text {wall }}$ is the catalyst surface area. The surface adsorption/desorption rates for each $i^{\text {th }}$ species are obtained as:

$$
\dot{s}_{i}^{\prime \prime}=\sum_{k=1}^{3}\left(\nu_{k i}^{\prime}\left(r_{k}\right)-\nu_{k i}^{\prime \prime}\left(r_{k}\right)\right),
$$

where the summation is over the three chemical reactions in the reduced reaction mechanism. The expressions for the reaction rates and the chemical reaction mechanism are explained in the next section.

The boundary conditions represented by equations 6 are incorporated in the present one-dimensional finite volume model by adding them as a source term in the species mass and energy equations. The corresponding source terms can be obtained as

$$
\begin{aligned}
\dot{s}_{i}^{\prime \prime \prime} & =\frac{\dot{s}_{i}^{\prime \prime} \mathcal{M}_{i} A_{c v}}{\mathcal{V}_{c v}} \\
\dot{q}_{\text {surf }}^{\prime \prime \prime} & =\frac{\dot{q}_{\mathrm{surf}}^{\prime \prime} A_{c v}}{\mathcal{V}_{c v}}
\end{aligned}
$$

where $\mathcal{V}_{c v}$ and $A_{c v}$ are the volume of the computational cell and surface area of the catalyst within the computational cell.

The coupled equations for mass, species concentrations, and energy are solved using an iterative successive over-relaxation scheme. The species advection terms are discretized using a second-order Beam-Warming scheme, properties at the fluxes are evaluated using simple arithmetic averages, and diffusive fluxes are approximated using a piecewise linear profile assumption (resulting in a symmetric central differencing). Solution is obtained within only a few seconds on a laptop.

\subsection{Chemical Kinetics for Heterogeneous Reactions}

In the microchannel-based solar reactor, chemical reactions can occur in the gaseous phase as well as in a series of reactions on the catalyst surface. Past 
studies by Deutschmann \& Schmidt [15] on oxidation of steam in a tubular model showed that for atmospheric pressures, the gas-phase reactions contributed negligibly to the oxidation process In the present work, experiments are conducted at atmospheric conditions and the gas-phase reactions are neglected.

It should be noted that the predictive capability of the numerical approach depends on accurate characterization of the surface reactions rates. Detailed chemical kinetics pathways for catalytic surface reactions $[16,15]$ were used in the modeling of the catalytic reaction on the channel surface. Kuznetsov and Koslov [17] used the same three-step reactions, however modeled the reaction rates using the expressions developed by Hou and Hughes [18], Xu and Froment [19] based on packed-bed reactor studies.

The catalytic reaction rates are nonlinear relations comprising the reactant species concentrations and the local temperature. Modeling of detailed chemical kinetics pathways for catalytic reactions on the surface have been performed [16, 15]; however, it can become expensive for wide range of parametric simulations performed in the present work. A reduced reaction mechanism with the following two endothermic (equations 10,12) and one exothermic water-gas shift (equation 11) global reactions was used to model the chemical conversion. The heats of reaction given for each equation is at $298 \mathrm{~K}$. To calculate the reaction rates, the classical kinetic model was employed $[20,21]$.

$$
\begin{array}{rll}
\mathrm{CH}_{4}+\mathrm{H}_{2} \mathrm{O} & \Longleftrightarrow \mathrm{CO}+3 \mathrm{H}_{2} ; & \Delta H_{R}^{0}=+206 \mathrm{~kJ} / \mathrm{mol} \\
\mathrm{CO}+\mathrm{H}_{2} \mathrm{O} & \Longleftrightarrow \mathrm{CO}_{2}+\mathrm{H}_{2} ; & \Delta H_{R}^{0}=-41 \mathrm{~kJ} / \mathrm{mol} \\
\mathrm{CH}_{4}+2 \mathrm{H}_{2} \mathrm{O} & \Longleftrightarrow \mathrm{CO}_{2}+4 \mathrm{H}_{2} ; & \Delta H_{R}^{0}=+165 \mathrm{~kJ} / \mathrm{mol} .
\end{array}
$$

There have been previous studies on methane-steam reforming in minichannels [22] using similar three-step reduced reaction mechanism with Arrheniustype reaction rates. As a validation against literature, the numerical approach together with the Arrhenius-type surface reaction rate model [11] were validated against the numerical studies on micrchannel methane-steam reforming performed by Kuznetsov and Koslov [17] using a Nickel catalyst. Their work incorporates the commonly used reaction rate model based on packed-bed reactor experiments by Hou and Hughes [18].

As mentioned earlier, the catalyst used here was a porous felt with deposited palladium nanoparticles throughout the felt. In this work, chemical kinetics characteristics for palladium catalyst are taken from Shu et al. [23]. In order to account for this distribution in the numerical model, it 
was necessary to quantify the surface distribution of the deposited Palladium catalyst. However, since the bed was porous, determining the number and location of active catalyst sites and its effect on reaction rates was not straightforward. Hence, a reaction-rate correction factor, $\alpha_{s}$, that modifies the pre-exponential factor in the Arrhenius reaction rates (equation 13) was therefore introduced. Experimental data were used to obtain this reactonrate correction factor as explained in the results section. Thus, the reaction reaction rates for each chemical reactions was formulated as

$$
\begin{aligned}
r_{k} & =k_{f, k} \Pi_{i=1}^{N_{s}}\left[\mathrm{C}_{i}\right]^{\nu_{k i}^{\prime}}, \\
k_{f, k} & =\alpha_{s} A_{k} \exp \left(-\frac{E_{a, k}}{R T}\right) .
\end{aligned}
$$

where $\mathrm{C}_{i}$ denotes the concentration per unit volume of the $i^{\text {th }}$ chemical species in the mixture, $k_{f, k}$ is the specific reaction rate constant for the $k^{t h}$ reaction and $\nu_{k i}^{\prime}$, which is dimensionless, is the stoichiometric coefficient of the $i^{\text {th }}$ chemical species in the $k^{t h}$ reaction. The reaction activation energy $\left(E_{a, k}\right)$ and the activation constants $A_{k}$ for each reaction are obtained from the experimental data $[18,19]$ for Palladium are given in Table 1 . The reaction-rate correction factor $\alpha_{s}$ is obtained from present experimental data as described later.

Table 1: Pre-exponential factors and activation energies (in $\mathrm{kJ} / \mathrm{mol}$ ) for the three reactions (equations 10,11,12) for Palladium catalyst.

\begin{tabular}{ccccccc}
\hline Catalyst & $A_{1}$ & $A_{2}$ & $A_{3}$ & $E_{a, 1}$ & $E_{a, 2}$ & $E_{a, 3}$ \\
\hline Palladium [23] & $89.6 \times 10^{9}$ & 543.5 & $371.1 \times 10^{9}$ & 109.4 & 15.4 & 209 \\
\hline
\end{tabular}

\section{NUMERICAL RESULTS}

This section first discusses how the reaction rate correction factor for the numerical model was obtained by performing calibration studies based on matching the exit hydrogen concentrations with experimental data $[1,2]$. The 1D model with appropriate reaction rate correction factor is then used at other experimental conditions for validation. The validated model is then used to perform parametric studies varying net heat flux, heat flux distribution, and inlet flow rates to guide in establishing operating parameters for maximal production of hydrogen. Finally, the shape-constrained optimization procedure and coupling to MONT2D radiative heat solver for obtaining the shape of the receiver is described. 


\subsection{Quantification of the Reaction-Rate Correction Factor}

The quantification of the reaction-rate correction factor using representative experimental data is described here. For specified inlet flow rates, steamto-methane ratio and net heat flux into the reactor, the $\alpha_{s}$ value was varied in the 1-D model to match the bulk exit molar concentration for $H_{2}$ in the experiments. The bulk mass fraction (and concentration) at a cross-section is obtained as:

$$
Y_{b u l k, H_{2}}=\frac{1}{A_{c} \bar{u}} \int_{A_{c}} u Y_{H_{2}} d A .
$$

where $\bar{u}$ is the average velocity at the cross-section of area $A_{c}$. Owing to the heat losses in the experiments, the heat fluxes going into each segment of the microchannel reactor were not available; rather the total heat input into the reactor was obtained from experiments by doing an overall energy balance. The heat input was assumed uniformly distributed along the microchannel surface.

Table 2: Calibration studies to obtain reaction-rate correction factors by matching the exit hydrogen concentrations with experimental data [1].

\begin{tabular}{ccccccc}
\hline \hline Case & $\begin{array}{c}\mathrm{CH}_{4} \text { Flow } \\
\text { Rate } g / \text { min }\end{array}$ & $\begin{array}{c}\mathrm{H}_{2} \mathrm{O}: \mathrm{CH}_{4} \\
\text { Ratio }\end{array}$ & $\begin{array}{c}\text { Inlet } \\
\text { Vel. } \mathrm{m} / \mathrm{s}\end{array}$ & $\begin{array}{c}\text { Inlet } \\
\text { Temp. }\end{array}{ }^{\circ} \mathrm{C}$ & $\begin{array}{c}\text { Heat Flux } \\
\mathrm{W} / \mathrm{m}^{2}\end{array}$ & $\begin{array}{c}\text { Computed } \\
\alpha_{s}\end{array}$ \\
\hline 1 & 0.21 & 2.91 & 4.91 & 714 & 276.3 & 0.00004325 \\
2 & 0.203 & 2.96 & 5.09 & 761 & 399 & 0.0000612 \\
3 & 0.195 & 3.06 & 5.18 & 798.7 & 840.7 & 0.0000748 \\
4 & 0.188 & 3.25 & 5.38 & 836.9 & 1882 & 0.0001008 \\
5 & 0.183 & 2.87 & 5.06 & 902 & 3908 & 0.0001463 \\
\hline
\end{tabular}

Table 3: Exit hydrogen concentration compared to experimental results for catalyst $\mathrm{C}$ [1] with a constant reaction rate correction factor of $\alpha_{s}=$ $6.6 \times 10^{-5}$.

\begin{tabular}{ccccc}
\hline \hline Case & $\begin{array}{c}\text { Methane Flow Rate } \\
g / \text { min }\end{array}$ & $\begin{array}{c}\text { Experiments } \mathrm{H}_{2} \\
\text { Molar Fraction }\end{array}$ & $\begin{array}{c}\text { Predicted } \mathrm{H}_{2} \\
\text { Molar Fraction }\end{array}$ & Error \\
\hline 1 & 0.21 & 0.0788 & 0.1066 & $35.28 \%$ \\
2 & 0.203 & 0.1433 & 0.1488 & $3.84 \%$ \\
3 & 0.195 & 0.219 & 0.2039 & $6.89 \%$ \\
4 & 0.188 & 0.333 & 0.2831 & $14.98 \%$ \\
5 & 0.183 & 0.4803 & 0.4068 & $15.30 \%$ \\
\hline
\end{tabular}


Table 2 shows the different cases investigated and the corresponding reaction rate correction factors. Ideally, for all cases studied, the correction factor would remain constant; however, as seen from the Table 2, some variation in $\alpha_{s}$ value is observed, showing a monotonous decrease with increase in net heat input to the reactor.Averaging the $\alpha_{s}$ values based on the representative cases shown in Table 2 , the mean reaction rate correction factor was obtained to be $6.6 \times 10^{-5}$, indicating that the active catalyst sites were a small fraction, consistent with the visual observations in the SEM images. Once this averaged reaction-rate correction factor was determined, it was kept constant and all the cases were run again to establish the errors in numerically predicted exit hydrogen concentrations compared to experimental measurements. Table 3 indicates the errors in the predicted hydrogen concentrations compared to the experimentally measured values. Here, the dry concentrations (after removal of the steam for gas chromatography) are compared showing predictions with reasonable errors. Errors less than $16 \%$ were observed for all cases except one with low heat input. These errors are within the experimental uncertainty given considerable heat losses observed in the experimental facility [1].

\subsection{Parametric Studies Investigating $\mathrm{H}_{2}$ Production}

The validated $1 \mathrm{D}$ model was used to perform parametric studies varying the net heat input to the reactor, the heat flux profile (constant, ramp up, ramp down) along the channel, and the inlet flow rates. Only representative results are shown here owing to space constraints.

\subsubsection{Net Heat Input}

The effect of external heat flux on $\mathrm{H}_{2}$ production was investigated as the reaction rates are highly dependent on the local surface temperature. Three heat flux magnitudes were enforced at mean values of 2,3 and $4 \mathrm{~kW} / \mathrm{m}^{2}$ with uniform distribution along the channel wall. The corresponding amount of heat input was $394.5,450.9$ and $511.05 \mathrm{~W}$, respectively. The steam-methane ratio was set to 2.7 (case $\mathrm{C} 7$ ) and the flow rate at $0.183 \mathrm{~g} / \mathrm{min}$. With increase in the amount of heat flux, the $\mathrm{H}_{2}$ production as well as reaction rates at the

wall increased. Increased value of heat flux increases the temperature along the wall, leading to enhanced $\mathrm{H}_{2}$ production. The average temperatures for these cases over the reactor were $1145.7,1170.3$ and $1194.3 \mathrm{~K}$, respectively (see figure 4a).

The reaction rates (for R3) plot (figure $4 \mathrm{~b}$ ) showed maximum reaction near the entrance with continuous decrease in the reaction rate along the channel. The overall reaction rate values were higher for the higher heat 

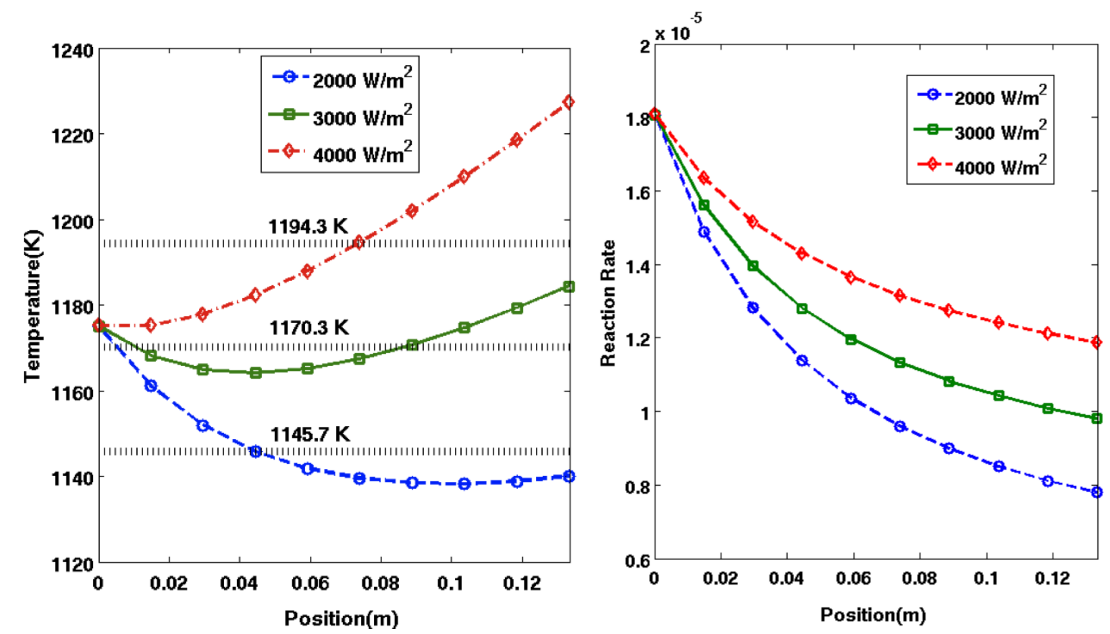

Figure 4: Distributions along the reactor for different uniform heat flux distributions: (a) temperature $(\mathrm{K})$, (b) reaction rate $\left(\mathrm{kmol} / \mathrm{m}^{2}-\mathrm{s}\right)$ for reaction 12. Also shown are the corresponding average reactor temperatures in dashed lines.

flux input. Production of $\mathrm{H}_{2}$ is an endothermic reaction, and this causes the temperature at the wall to initially decrease. Reactions continue to occur along the channel wall, however, their rate is decreased. For all cases, the reaction rates went down after the mid-section, and the temperature increased owing to thermal heating of the gases. For the cases studied, only $26.9 \%$ of the initial methane was converted to hydrogen, which is similar to the experimental observation. This may be attributed to small residence times compared to the reaction time scales as well as non-uniformity of reaction sites on the porous surface.

\subsubsection{Heat Flux Profile}

In practical solar receivers, it is expected that the heat flux profile along the microchannel wall be non-uniform. In order to explore the effect of heatflux profile on the non-linear nature of the reaction rates and $\mathrm{H}_{2}$ production, three different heat flux profiles were considered: uniform distribution, ramp up, and ramp down wherein the heat flux is varied linearly along the channel walls. To study the effect of heat flux profile, the average heat input over the microchannel length was kept fixed and the steam-methane ratio was set to 2.7 and the flow rate at $0.183 \mathrm{~g} / \mathrm{min}$. Three different values of the net heat input of $394.5,450.9$ and $511.05 \mathrm{~W}$ were studied. 

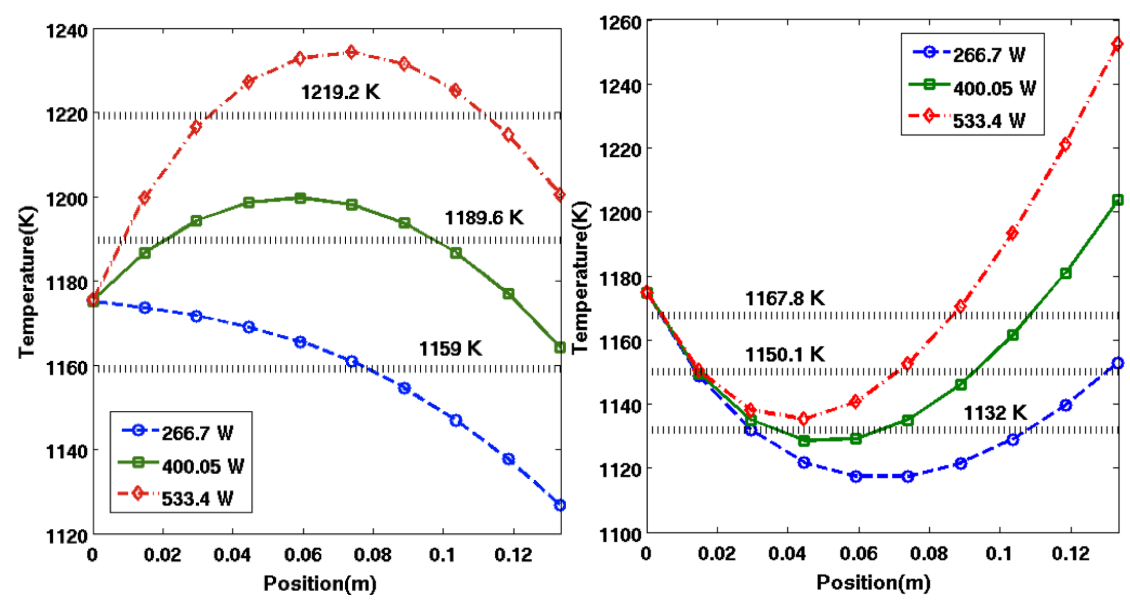

Figure 5: Temperature distribution along the reactor for different heat flux distributions: (a) linearly descreasing, (b) linearly increasing. Also shown are the corresponding average reactor temperatures.

The temperature distributions along the reactor for different heat flux distributions, uniform, linearly decreasing, and linearly increasing are shown in figures $4 \mathrm{a}, 5 \mathrm{a}$ and $5 \mathrm{~b}$, respectively. The most interesting case is that of the decreasing heat flux profile. Figure 5a shows the variation of catalyst temperature along the reactor for three different net heat inputs. It is observed that the mean reactor temperatures were larger for this case compared to the uniform and decreasing ramp heat flux distributions. For the highest heat input of $533.4 \mathrm{~W}$, the temperature of the reactor actually increases in the first half of the channel and then decreases. Since endothermic reactions are occurring in the first half, having larger heat flux (decreasing ramp), seems to benefit the reaction rates and net exit hydrogen production. This suggests that using decreasing ramp for heat flux distribution is important and makes use of the input heat for actual methane conversion rather than thermal heating of the product gases as observed in the uniform and increasing heat flux profiles. The hydrogen production (based on exit molar fraction) for the decreasing heat flux profile was almost $25 \%$ higher compared to the increasing ramp function and $11 \%$ higher compared to the uniform heat flux profile for the net heat input of $533.4 \mathrm{~W}$.

\subsubsection{Inlet Flow Rate}

For the baseline case studied (case $\mathrm{C} 7$ ) as well as in the experiments, the inlet flow rate of the methane-steam mixture was $0.183 \mathrm{~g} / \mathrm{min}$ and the cor- 
responding mean inlet velocity is $5.06 \mathrm{~m} / \mathrm{s}$. This, however, resulted in partial conversion of the methane to hydrogen. The molar conversion of a reactant flowing at a fixed velocity in a heterogeneous chemical reactor of fixed length is dictated by diffusion $\left(\tau_{\mathrm{d}, \mathrm{i}}=(\mathrm{H} / 2)^{2} / \mathcal{D}_{i m}\right)$ and reaction $\left(\tau_{\text {kin }}=1 / k\right)$ time scales, the ratio of which is embodied in the Damkohler number $\left(\mathrm{D} a=\tau_{\mathrm{d}, \mathrm{i}} / \tau_{\text {kin }}\right)$ [24]. Here $\mathrm{H}$ is the channel height; $\mathcal{D}_{i m}$ is the diffusion coefficient of species $i$ in mixture $m$, and $k$ is the reaction rate constant. By measuring the product concentrations, an estimate of the conversion of the reactants to products can be obtained. This conversion is affected by diffusion as well as reaction rates and residence times. In addition, the residence time of reactants inside the microchannel is critical and is given by $\tau_{\text {res }}=L / \bar{u}$, where $L$ is the length of the microchannel and $\bar{u}$ is the mean inlet velocity. For the baseline case $(\mathrm{C} 7)$, the residence time is $0.0263 \mathrm{~s}$, whereas the diffusion time scale is $0.001038 \mathrm{~s}$. This resulted in methane conversion of only $26.9 \%$ based on the inlet and exit molar fractions for the highest heat input.
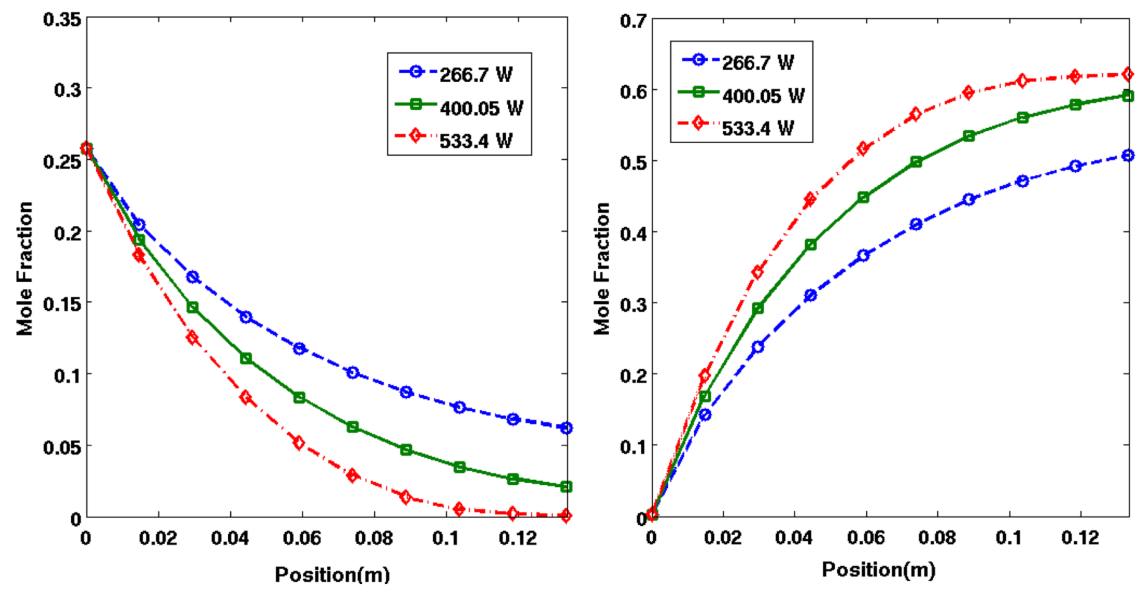

Figure 6: Mole fraction distributions along the reactor for linearly decreasing heat flux distribution with an inlet flow rate of $0.026 \mathrm{~g} / \mathrm{min}$ : (a) $\mathrm{CH}_{4}$ and (b) $\mathrm{H}_{2}$

A series of simulations were performed varying the inlet flow rates till complete conversion of methane to hydrogen was obtained, keeping all other parameters the same. The inlet mean flow velocity for complete conversion was found to be $0.721 \mathrm{~m} / \mathrm{s}$ corresponding to a flow rate of $0.026 \mathrm{~g} / \mathrm{min}$ (figures $6 \mathrm{a}, \mathrm{b})$. The corresponding residence time is $0.185 \mathrm{~s}$. With increased residence times, it was observed that the reaction rates for the endothermic 
reaction 11 steadily increased from the inlet section till the outlet section, resulting in complete conversion. This indicates that for complete conversion, a longer channel (almost five times) would be necessary at the same baseline flow rate used in experiments.

\subsection{Receiver Shape Optimization}

The hydrogen produced by a solar receiver will depend upon the radiative heat transfer occurring within the receiver that results in a heat flux profile along the receiver walls and the reactions occurring within the microchannels. The shape of the receiver dictates the incident radiative heat flux profile. Ideally, shape optimization of the receiver would entail specifying an initial receiver shape, with Monte-Carlo based radiative heat transfer and ray tracing employed to determine a heat flux profile along the length of the receiver. This heat flux profile would then be employed in a 1-D steady reduced-reaction model to determine the steady state hydrogen output for the receiver shape. In absence of the fully coupled receiver shape-reactor modeling approach to predict hydrogen output, receiver shape optimization is limited in this work to matching a desired heat-flux profile along the length of the receiver. To illustrate the methodology and present approach, a linear heat flux variation along the length of the receiver was used. As shown previously, a decreasing ramp function for the heat flux profile along the microchannel provides the most hydrogen output. As such, the optimization problem examined in this paper reduces to identifying a receiver shape that explicitly matches a prescribed linearly varying heat flux profile along the length of the receiver wall.

In this work, the shape of the solar receiver is assumed symmetric about an axis of revolution, such that the receiver can be designed in two dimensions $(r, z)$. Reactants are assumed to enter at the cavity opening side of the receiver and flow along the length of the receiver within microchannel reactors and exit at the rear end of the receiver. The rear of the receiver is assumed to consist of a flat bottom of radius $r_{0}$, located on the $r$-axis, and the receiver extends for a height $h$ along the $z$-axis. As stated, the optimization search space contains the set of all possible receiver shapes. To reformulate the maximization problem in a finite, lower-dimensional form that can be numerically solved by sequential quadratic programming (SQP) algorithms, the receiver shape is instead represented by a set of coordinates $\left(r_{i}, z_{i}\right)$. Chebyshev polynomials are utilized to fit a receiver shape through these points, where $r_{i}$ represent the radial position of the Chebyshev interpolation points. In this manner, the optimization problem is therefore trans- 


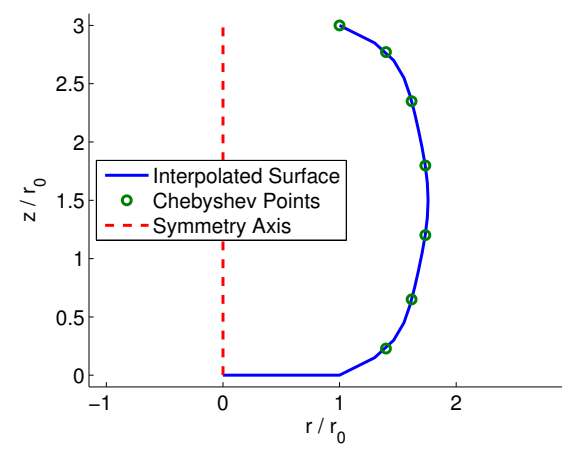

Figure 7: Example of axisymmetric receiver shape. The symbols are the Chebyshev points that define the shape. 20 discrete surfaces are fit to the Chebyshev polynomial to form the enclosure sent to MONT2D.

formed into one in which the optimization routine must determine the coordinates $r_{i}$ at each grid point such that a quadratic cost function $J=\mathrm{z}^{T} \mathbf{Q z}$ is minimized. The minimization of the cost function is performed subject to design constraints on the radial locations of the interpolation points. In the cost function $J$, the vector $\mathrm{z}$ represents the difference between the heat flux produced by the receiver shape and the desired heat flux profile at prescribed locations along the receiver length and $\mathbf{Q}$ represents a weighting matrix.

Although finite in number, the coordinates $\left(r_{i}, z_{i}\right)$ produce a continuous receiver shape via the Chebyshev polynomial representation. The resulting constrained, finite dimensional, nonlinear optimization problem is solved in MATLAB using SNOPT [25], a particularly robust implementation of a SQP algorithm. Provided with an initial guess of the receiver shape, as specified by the radial locations of the Chebyshev interpolation points, a MATLAB function discretizes the receiver shape via piecewise linear polynomials for use in MONT2D, a freely available Monte-Carlo based radiative heat transfer and ray tracing program [12]. These studies use seven Chebyshev points to create the interpolated polynomial which is discretized into 20 surfaces for MONT2D. Figure 7 shows an example of the Chebyshev surface interpolation and surface discretization for an arbitrary receiver shape. The MATLAB function creates an input file with the coordinates of the discretized receiver shape along with material properties and solver options before starting the MONT2D solver.

MONT2D interprets the radial position of the last Chebyshev point as the radius of the aperture through which solar energy enters the receiver. 
MONT2D requires a surface to be specified at the aperture so that all of the surfaces form an enclosure. At the aperture surface, MONT2D emits and tracks photons, through possible reflections, until absorption. The aperture is assumed to be a diffuse emitter and a blackbody absorber as all the photons that hit the aperture surface would be lost to the surroundings. The receiver walls are assumed to be a stainless steel that is a perfectly diffuse reflector and absorbs $50 \%$ of incoming photons independent of incident angle. Additionally, all surface properties are assumed to be independent of radiation wavelength. The exchange fraction is defined by $F_{i}=N_{i} / N_{\text {total }}$, where $F_{i}$ is the exchange fraction between the aperture surface and surface $i, N_{i}$ is the total number of photons absorbed by surface $i$, and $N_{\text {total }}$ is the total number of emitted photons from the aperture surface. The MATLAB code reads the output file from MONT2D and proceeds to calculate a heat flux profile. The total heat flux is specified for the aperture and thus the total radiative energy entering the enclosure is known. The incident radiation for a given surface is given by $q_{i}=q_{\text {aperture }} * A_{\text {aperture }} * F_{i} / A_{i}$, where $q_{i}$ is the energy flux absorbed by surface $i, q_{\text {aperture }}$ is the energy flux entering the aperture, $A_{\text {aperture }}$ is the axisymmetric area of the aperture, $F_{i}$ is the exchange fraction between the aperture surface and surface $i$, and $A_{i}$ is the axisymmetric area of surface $i$. The $q_{i}$ values form a discrete heat flux profile along the length of receiver wall accounting for the length changes of the receiver based on the wall curvature. This profile is compared to the target linear profile for each surface and the error vector, $z$, is calculated as the absolute value of the difference between the calculated heat flux and the target heat flux normalized by the number of surfaces. For these studies the weighting matrix $\mathbf{Q}$ was the identity matrix.

SNOPT was subsequently responsible for determining new values for the radial locations of the Chebyshev interpolation points and this process was repeated until the cost function, subject to the bounds on the values for the interpolation points, was minimized. The efficacy of this strategy is illustrated through its ability to identify a receiver shape that produced a ramp-down heat flux profile that varied linearly along the length of the receiver. The optimized shape is shown in figure 8 . The heat flux profile is independent of length scale and scales linearly with the aperture heat flux for a constant aspect ratio.

These results show that it is possible to closely match a linear heat flux profile with a combination of SNOPT and MONT2D. The average and maximum error between the calculated and target heat flux profiles are $1.2 \%$ and $11.9 \%$ respectively. While this final error is small, there are two factors that reduce the robustness of this method. Appropriate values require an itera- 


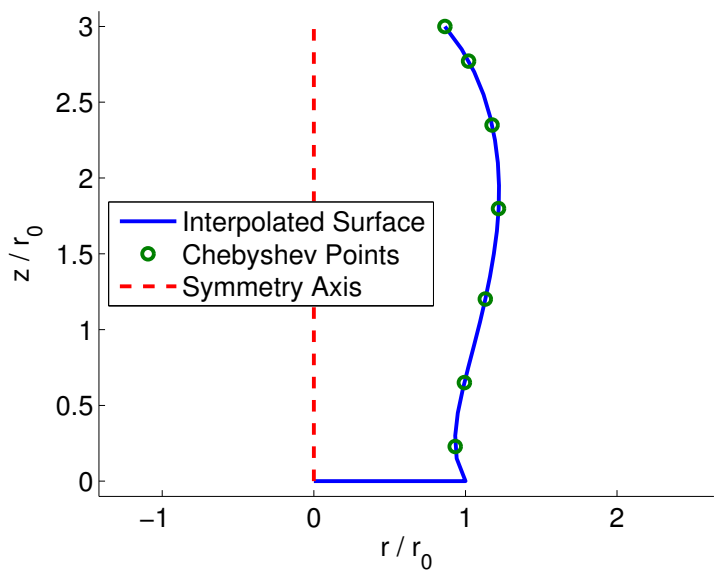

Figure 8: Receiver shape obtained from the optimization procedure for a specified ramp-down heat flux profile. Here the reactants are flowing from $z / r_{0}=0$ to $z / r_{0}=3$.

tive approach by the user. Additionally, the precision of Monte-Carlo results somewhat interferes with the SNOPT optimization routine. This routine works best with smooth or nearly-smooth functions. By their stochastic nature, Monte Carlo methods do not produce smooth results. Using the same inputs, exec6uting MONT2D twice will produce slightly different answers. This difference reduces robustness especially when SNOPT estimates derivatives of the objective function, $J$, using finite difference approximations. To mitigate this effect, the number of photons emitted by MONT2D was increased to a value on the order of $10^{8}$. This significantly increased the computational expense of evaluating $J$, but was necessary for SNOPT to find an acceptable solution.

\section{CONCLUSION}

The design of a microchannel reactor geometry for methane-steam reforming was investigated using a simple steady-state one-dimensional model . Methane-steam reforming was modeled by three reduced-order reactions occurring on the reactor walls, two of which were endothermic reactions and an exothermic water-gas shift reaction. The surface chemical reactions were modeled source terms in the energy and species mass fraction equations, with Arrhenius-type reaction rate models for Palladium-deposited catalyst.

First the numerical model was applied to predict hydrogen production in 
a microchannel setup based on the experimental setup of Eilers [1] to show good predictive capability. In order to account for the non-uniformity and intermittency in active sites, a simple model modifying the reaction rate constant through a single constant factor was investigated. The reaction-rate correction factor was determined by matching the exit hydrogen production against the experimental data for one particular flow rate. Using this reaction-rate constant, simulations were performed at different flow rates and hydrogen production was compared with the experimental data to show reasonable agreement. This validation shows that use of an experimentally calibrated reaction-rate factor can be used to perform parametric studies and prediction of hydrogen production in microchannel reactors. Parametric studies were then performed by varying the net heat input, heat flux profile along the microchannel, and the inlet flow rate to study their effect on the methane conversion.For a given flow rate and heat input, a decreasing ramp function for the heat flux along the microchannel yielded increased hydrogen production. By controlling the inlet flow rates that alter the residence time compared to the reaction time scales, complete conversion of $\mathrm{CH}_{4}$ to $\mathrm{H}_{2}$ was feasible.

Finally, a shape-constrained optimization procedure using the SNOPT algorithm coupled with the radiative heat transfer module MONT2D [12] was developed to design the receiver shape that provides a desired heat flux profile along the microchannel length. This optimization process can be used to design receiver shapes for optimal hydrogen production.

\section{Acknowledgement}

This work was funded partly by the Oregon Best Solar energy initiative and the Oregon Nano and Microtechnology Institute (ONAMI). We also thank Prof. Murty Kanury of OSU for several useful discussions related to combustion and chemical kinetics modeling.

\section{References}

[1] Eilers, B., 2010, "Microchannel steam-methane reforming under constant and variable surface temperature distributions," M.S. Thesis, Oregon State University.

[2] Eiler, B., Narayanan, V., Apte, S., and Schmitt, J., 2011, "Steammethabe reforming in a microchannel under constant and variable surface temperature profiles," AJTEC2011-44390, Proceedings of the 
ASME/JSME 2011 8th Thermal Engineering Joint Conference, Honolulu, Hawaii, USA.

[3] Tamme, R., Buck, R., Epstein, M., Fisher, U., and Sugarmen, C., 2001, "Solar upgrading of fuels for generation of electricity," Journal of Solar Energy Engineering, 123, p. 160.

[4] Möller, S., Kaucic, D., and Sattler, C., 2006, "Hydrogen production by solar reforming of natural gas: a comparison study of two possible process configurations," Journal of Solar Energy Engineering, 128, p. 16.

[5] Kodama, T., Moriyama, T., Shimoyama, T., Gokon, N., Andou, H., and Satou, N., 2006, "Ru/ Ni-Mg-O catalyzed SiC-foam absorber for solar reforming receiver-reactor," Journal of Solar Energy Engineering, 128, p. 318.

[6] Wegeng, R., TeGrotenhuis, W., and Mankins, J., 2007, "Solar thermochemical production of fuels," AIAA, 2007-4709, 5th International Energy Conversion Engineering Conference and Exhibit.

[7] Wegeng, R. and Mankins, J., 2009, "Space power systems: Producing transportation (and other chemical) fuels as an alternative to electricity generation," Acta Astronautica, 65(9-10), pp. 1261-1271.

[8] Steinfeld, A., 2005, "Solar thermochemical production of hydrogen--a review," Solar Energy, 78(5), pp. 603-615.

[9] Röger, M., Pfander, M., and Buck, R., 2006, "Multiple air-jet window cooling for high-temperature pressurized volumetric receivers: Testing, evaluation, and modeling," Journal of Solar Energy Engineering, 128(3), pp. 265-274, URL http://link. aip.org/link/?SLE/128/265/1.

[10] Kraupl, S. and Steinfeld, A., 2001, "Pulsed gas feeding for stoichiometric operation of a gas-solid vortex flow solar chemical reactor," Journal of Solar Energy Engineering, 123, p. 133.

[11] Drost, K., Eiler, B., Peterson, D., Apte, S., Narayanan, V., and Schmitt, J., 2011, "Detailed numerical modeling of a microchannel reactor for methane-steam reforming," AJTEC2011-44664, Proceedings of the ASME/JSME 2011 8th Thermal Engineering Joint Conference, Honolulu, Hawaii, USA. 
[12] Maltby, J., Zeeb, C., Dolaghan, J., and Burns, P., 1994, "Users manual for MONT2D-version 2.6 and MONT3D-version 2.3," Department of Mechanical Engineering, Colorado State University, Fort Collins, CO.

[13] Valmikanathan, O., Ostroverkhova, O., Mulla, I., Vijayamohanan, K., and Atre, S., 2008, "The effect of synthesis procedure on the structure and properties of palladium/polycarbonate nanocomposites," Polymer, 49(16), pp. 3413-3418.

[14] Brust, M., Bethell, D., Kiely, C., and Schiffrin, D., 1998, "Selfassembled gold nanoparticle thin films with nonmetallic optical and electronic properties," Langmuir, 14(19), pp. 5425-5429.

[15] Deutschmann, O. and Schmidt, L., 1998, "Modeling the partial oxidation of methane in a short-contact-time reactor," AIChE Journal, 44(11), pp. 2465-2477.

[16] Stutz, M. J. and Poulikakos, D., 2005, "Effects of microreactor wall heat conduction on the reforming process of methane," Chemical Engineering Science, 60(24), pp. $6983-6997$.

[17] Kuznetsov, V. and Kozlov, S., 2008, "Modeling of methane steam reforming in a microchannel with a heat flow distributed in length," Journal of Engineering Thermophysics, 17(1), pp. 53-59.

[18] Hou, K. and Hughes, R., 2001, "The kinetics of methane steam reforming over a $\mathrm{Ni} / \alpha-\mathrm{Al} 2 \mathrm{O}$ catalyst," Chemical Engineering Journal, 82(1-3), pp. 311-328.

[19] Xu, J. and Froment, G., 1989, "Methane steam reforming, methanation and water-gas shift: I. Intrinsic kinetics," AIChE Journal, 35(1), pp. $88-96$.

[20] Kenneth, K., 2005, "Principles of combustion," John Wiley's sons, Ine, Hoboken, New Jersey.

[21] Turns, S., 1995, "An introduction to combustion: Concepts and applications(Book)," New York: McGraw-Hill, Inc, 1995.

[22] Wang, Y., Yoshiba, F., Kawase, M., and Watanabe, T., 2009, "Performance and effective kinetic models of methane steam reforming over $\mathrm{Ni} / Y S Z$ anode of planar SOFC," International Journal of Hydrogen Energy, 34(9), pp. 3885-3893. 
[23] Shu, J., Grandjean, B., and Kaliaguine, S., 1994, "Methane Steam Reforming in Asymmetric Pd-Ag and Pd-Ag/Porous SS Membrane Reactors," Applied Catalysis A: General, 119(2), pp. 305-325.

[24] Kanury, A., 1984, "Introduction to combustion phenomena," Gordon and Breach Science Publishers, New York, Fourth printing.

[25] Gill, P., Murray, W., and Saunders, M., 2005, "SNOPT: An SQP algorithm for large-scale constrained optimization," SIAM Review, 47(1), pp. $99-131$. 